\title{
Estimativas do Consumo e da Taxa de Passagem do Capim-Elefante (Pennisetum purpureum Schum.) sob Pastejo de Vacas em Lactação 1
}

\author{
João Paulo Guimarães Soares ${ }^{2}$, Luiz Januário Magalhães Aroeira ${ }^{3,6}$, Rui da Silva Verneque ${ }^{3,6}$, \\ Odilon Gomes Pereira ${ }^{4,6}$, Carlos Eugênio Martins ${ }^{3,6}$, Sebastião de Campos Valadares Filho ${ }^{4,6}$, \\ William José Ferreira ${ }^{5}$
}

\begin{abstract}
RESUMO - Foram estimados o consumo de matéria seca (MS), a taxa de passagem e o tempo médio de retenção no trato gastrintestinal de 18 vacas Holandês-Zebu, em lactação, pastejando capim-elefante. Nas estimativas de consumo e da produção fecal usaram-se os modelos propostos por QUIROZ et al. (1988) e POND et al. (1989). Para as taxas de passagem e tempos de retenção, incluiu-se a equação proposta por GROVUM e WILLIAMS (1973). Nas avaliações de consumo, utilizaram-se piquetes de capim-elefante, com três dias de ocupação e 30 de descanso, adubado com duas doses de nitrogênio ( 300 e $700 \mathrm{~kg} / \mathrm{ha} / \mathrm{ano}$ ), numa taxa de lotação de 6 vacas/ha, em quatro meses do ano. O consumo foi estimado pela relação produção fecal/indigestibilidade da forragem. Nas determinações da digestibilidade e da produção fecal foram usadas amostras de extrusas. Para a excreção fecal foi usada a fibra detergente neutra, tratada com dicromato de sódio. As estimativas do consumo de MS da dieta total e do capim-elefante não apresentaram diferenças $(\mathrm{P}>0,05)$ em função dos modelos utilizados. Registraram-se valores médios de consumo de 12,5 e 13,9 kg de MS/vaca/dia para a dieta total e 7,7 e 9,2 kg de MS/ vaca/dia do capim-elefante, respectivamente, pelos modelos de POND et al. (1989) e QUIROZ et al. (1988). As taxas de passagem, os tempos médios de retenção no rúmen e no trato gastrintestinal apresentaram diferenças, quando utilizados os três modelos testados. No presente experimento, optou-se pela adoção dos resultados obtidos pelo modelo de QUIROZ et al. (1988), para se estimar consumo, devido à semelhança dos dados com aqueles já descritos na literatura.
\end{abstract}

Palavras-chave: capim-elefante, consumo, cromo mordentado, modelos, taxa de passagem, vacas em lactação

\section{Estimates of Feed Intake and Rate of Passage of Elephantgrass (Pennisetum purpureum Schum.) under Grazing Conditions using Lactating Cows}

\begin{abstract}
The voluntary dry matter intake (DMI), rate of passage and mean retention time in the rumen and gastrointestinal tract were estimated using 18 crossbred Holstein-Zebu lactating cows grazing elephantgrass. The voluntary DMI and fecal output estimations the models proposed by QUIROZ et al. (1988) and POND et al. (1989) were used. The data of rate of passage and those of retention time in the rumen the model suggested by GROVUM and WILLIAMS (1973) was chosen. The elephantgrass pasture were fertilized and managed in a rotational system with three days paddock occupation and 30 days resting. The stocking rate was 6 cows/ha. The DMI was estimated using the relation fecal output/forage indigestibility of extrusa samples. For the fecal production calculations, neutral detergent fiber was chromium mordanted. Using the models of POND et al. (1989) and QUIROZ et al. (1988) to compare the total diet DMI (12.5 and $13.9 \mathrm{~kg} / \mathrm{cow} /$ day) and elephantgrass DMI ( 7.7 and $9.2 \mathrm{~kg} / \mathrm{cow} /$ day) they did not differ, respectively. However, the rate of passage and the mean retention time in the gastrointestinal tract did differ among the three used models. The results of this trial showed that the model proposed by QUIROZ et al. (1988) was the one that better describes the DMI data.
\end{abstract}

Key Words: chromium mordent, elephantgrass, intake, lactating cows, models, passage rate

\section{Introdução}

A estimativa indireta da ingestão de alimentos, principalmente de animais em pastejo, parece mais adequada do que as medidas diretas, mas na prática oferece inúmeras dificuldades relacionadas a distúrbios causados aos animais nas administrações das doses de indicadores inertes (OWENS e HANSON, 1992).

O consumo voluntário de animais em pastejo, geralmente, é determinado por meio de estimativas de digestibilidade (DIG) e produção fecal (PF) utilizando-se a relação: Consumo $=\mathrm{PF} /(1-\mathrm{DIG})$, em que a produção fecal pode ser determinada com o auxílio de indicadores externos e internos segundo POND et

\footnotetext{
1 Trabalho financiado pela Embrapa Gado de Leite.

2 Pesquisador da Embrapa Rondônia, Doutorando - FCAV/UNESP - Jaboticabal-SP. E-mail: jpsoares@fcav.unesp.br

3 Pesquisadores da Embrapa Gado de Leite. E.mail: laroeira@cnpgl.embrapa.br

${ }^{4}$ Professores do Departamento de Zootecnia da UFV. E.mail: odilon@mail.ufv.br

${ }^{5}$ Doutorando DZO/UFV. E.mail: wjferreira@lince.tdnet.br

${ }^{6}$ Bolsista do CNPq.
} 
al. (1994). Os indicadores são, geralmente, fornecidos aos animais a partir de infusões contínuas, administrações diárias ou a partir de uma dosagem única.

A administração de dose única de um indicador permite estimar a produção fecal, o consumo de matéria seca, a taxa de passagem da digesta, o tempo médio de retenção e o enchimento do trato gastrintestinal (POND et al., 1989; QUIROZ et al., 1988).

Sabe-se que a taxa de passagem do alimento no rúmen é uma variável importante na regulação do consumo. O maior ou menor tempo de retenção no retículo-rúmen influencia nos processos de digestão e de assimilação dos nutrientes. Segundo SEONE (1995), as forragens que ocuparam menor volume e que retiveram menos água foram consumidas em maior quantidade, devido, possivelmente, ao aumento da taxa de passagem. Além disso, o tamanho e a forma da partícula ingerida afetaram o consumo, a taxa de degradação e o tempo de retenção da digesta no rúmen (LUGINBUHL et al., 1991).

Alguns autores propuseram modelos para se estimar a taxa de passagem dos alimentos pelos diferentes segmentos do trato gastrintestinal (GROVUM e WILLIAMS, 1973; ELLIS et al., 1979; DHANOA et al., 1985; QUIROZ et al., 1988; POND et al., 1989).

Foram estudados diferentes modelos não-lineares para descrever a passagem de partículas de tamanhos diferentes e de líquidos pelo trato gastrintestinal de ruminantes. GROVUM e WILLIAMS (1973) utilizaram dois termos exponenciais como compartimentos de mistura e um terceiro compartimento, tubular, como tempo de trânsito e propuseram um método gráfico para calcular os parâmetros. Este modelo, embora seja o mais utilizado para as estimativas de taxa de passagem, é usado quando se considera o processo de passagem nos diferentes segmentos do trato digestivo como sendo independente do tempo de permanência nos diferentes compartimentos. Isto mostra que há igual probabilidade de escape para todas as partículas alimentares sem considerar o tamanho ou a idade.

Entretanto, ELLIS et al. (1979) propuseram modelos que consideram a probabilidade da passagem de partículas ser aumentada pelo tempo, ou seja, as taxas de passagem não permanecem constantes. Já DHANOA et al. (1985) consideraram a cinética da digesta como um processo exponencial multicompartimental, em que modelos foram utilizados para estimar a taxa de passagem por adequação da curva de excreção fecal, o número de compartimentos

Rev. bras. zootec., 30(6S):2183-2191, 2001 de passagem da digesta e a acurácia da predição.

QUIROZ et al. (1988) compararam alguns tipos de modelos como: $1^{\circ}$ ) modelo biexponencial de idade independente (GROVUM e WILLIAMS, 1973); $2^{\circ}$ ) modelo gama de idade-dependente (ELLIS et al., 1979) e $3^{\circ}$ ) variação dos modelos anteriores para se estimar as taxas de passagens no rúmen e pós-rúmen. Os modelos biexponenciais determinam que há uma igual probabilidade de escape de partículas, independentemente do tamanho ou idade. No entanto, nos modelos de idade dependente, a probabilidade de passagem das partículas aumenta com o tempo de permanência no trato gastrintestinal. Os autores sugeriram que, para forragens de fibras longas, o segundo modelo seria mais apropriado. Para dietas com diferentes tamanhos de partículas, a variação do segundo modelo, considerando idade dependente para o primeiro compartimento e independente para o segundo compartimento, seria o mais adequado.

O modelo de POND et al. (1989) é descrito como de um compartimento apenas, mas considera que não há uma igual probabilidade de escape para as partículas alimentares, sendo a passagem dependente do tamanho e da idade.

QUIROZ et al. (1988) observaram que o modelo que melhor descreve a cinética do trato gastrintestinal, sem levar em conta o tamanho de partícula, seria o mais apropriado devido a sua versatilidade, sendo este um modelo de dois compartimentos, que, contudo, considera para o primeiro compartimento (rúmenretículo) idade dependente e para o segundo (omasoabomaso), idade independente de passagem de partículas, sendo o utilizado no presente trabalho.

O objetivo deste estudo foi, além de avaliar o consumo de capim-elefante para vacas mestiças em lactação, comparar os valores estimados a partir dos modelos propostos por GROVUM e WILLIAMS (1973), QUIROZ et al. (1988) e POND et al. (1989).

\section{Material e Métodos}

O experimento foi realizado na Embrapa Gado de Leite, em Coronel Pacheco, Minas Gerais, durante o período de julho de 1996 a março de 1997.

O pasto de capim-elefante (Pennisetum purpureum, Schum.) cv. Napier foi estabelecido num Latossolo Vermelho-Amarelo, em janeiro de 1990, utilizando-se estacas, em sulcos distanciados de $50 \mathrm{~cm}$. Por ocasião do plantio, foram aplicados, no sulco, $100 \mathrm{~kg} /$ ha de $\mathrm{P}_{\mathbf{R}} \mathrm{O}_{5}$, utilizando-se o superfosfato 
simples. No início do experimento, aplicaram-se $300 \mathrm{e}$ $700 \mathrm{~kg} / \mathrm{ha}$ de $\mathrm{N}$, parcelados em três épocas, início meio e final do período chuvoso, utilizando-se a uréia no primeiro e o nitrocálcio no segundo ano, como fontes nitrogenadas. Junto de cada adubação nitrogenada, aplicaram-se $200 \mathrm{~kg} / \mathrm{ha}$ de $\mathrm{K}_{2} \mathrm{O}$, usando-se o cloreto de potássio como fonte de potássio.

Utilizou-se uma área de 3,0 ha, dividida em seis parcelas de 0,5 ha, que por sua vez foram subdivididas em 11 piquetes. O experimento foi montado no delineamento em blocos casualizados com três repetições. Cada piquete foi utilizado por um período de três dias, com 30 de descanso.

Foram utilizadas 18 vacas Holandês-Zebu, de segunda ou de terceira lactação, arranjadas por produção de leite na lactação anterior, cujos pesos médios variaram de 482 a $505 \mathrm{~kg}$ e as produções de leite de 9,8 a 14,3 kg/vaca/dia. Foram utilizadas nove vacas por tratamento, três em cada uma das repetições de área, permitindo uma taxa de lotação de seis vacas/ha.

Durante o período das chuvas, as vacas permaneceram na pastagem de capim-elefante, saindo apenas para ordenha. No período seco do ano, receberam suplementação volumosa à base de cana-de-açúcar, enriquecida com $1,0 \%$ de uréia, no intervalo das ordenhas, retornando à noite aos piquetes de capim-elefante.

A suplementação do concentrado foi feita por ocasião das duas ordenhas, na quantidade fixa de $2 \mathrm{~kg} /$ animal $/ \mathrm{dia}$. O concentrado apresentou a seguinte composição: milho-grão, $65 \%$; farelo de algodão, $10 \%$; farelo de trigo, $20 \%$; uréia, $2 \%$; calcário, $2 \%$; e mistura mineral, $1 \%$.

A estimativa da forragem disponível foi efetuada antes da entrada dos animais nos piquetes, segundo metodologia descrita por AROEIRA et al. (1996), utilizando-se a simulação de pastejo, em que foram escolhidas duas touceiras representativas de acordo com a disponibilidade alta e baixa e em dois pontos diferentes de cada piquete nas três repetições de cada tratamento. A seguir, retiraram-se das plantas toda a parte aérea, folhas verdes e caules tenros que eram colocados em sacos de papel, pesados e levados para secagem em estufa ventilada a $60^{\circ} \mathrm{C}$. O número de touceiras em cada piquete, numa área de $49 \mathrm{~m}^{2}$ foi contado. A disponibilidade de forragem foi estimada pela relação: Disponibilidade MS = Produção de MS (média de duas touceiras) x $\mathrm{N}^{\mathrm{O}}$ de touceiras em $49 \mathrm{~m}^{2}$.

Para a coleta de extrusas de cada piquete, utilizaram-se duas vacas mestiças Holandês-Zebu, secas, fistuladas no esôfago nos meses de julho, outubro, janeiro e março, antes da entrada dos animais experimentais nos piquetes.

Os animais não foram submetidos a jejum prévio para não influenciar o consumo, e as coletas foram feitas entre 8 e $11 \mathrm{~h}$, em três dos 11 piquetes de cada repetição de cada tratamento, seguindo-se a ordem de rotação dos mesmos. Coletaram-se as extrusas referentes ao segundo dia de pastejo de cada piquete, na quantidade de $3 \mathrm{~kg}$, para a confecção das amostras compostas dos três piquetes.

As amostras foram imediatamente congeladas a $-20^{\circ} \mathrm{C}$. Por ocasião do término de cada período, procedeu-se ao descongelamento dessas amostras, as quais foram divididas em duas porções: a primeira foi usada para determinação da digestibilidade in vitro da matéria seca (TILLEY e TERRY, 1963), e a segunda para o tratamento da fibra em detergente neutro (FDN) com dicromato de sódio $\left(\mathrm{Na}_{2} \mathrm{Cr}_{2} \mathrm{O}_{7} \cdot 2 \mathrm{H}_{2} \mathrm{O}\right)$, segundo metodologia descrita por COLUCCI (1984).

A produção fecal foi estimada, em 18 animais em pastejo (três animais de cada piquete), utilizando-se a FDN da extrusa mordentada com dicromato de sódio. O alimento marcado foi pesado e colocado em cápsulas de gelatina, com aproximadamente $5 \mathrm{~g}$. Cerca de 40 a $50 \mathrm{~g}$ desse material foi administrado a cada animal, em dose única, por via oral, com o auxílio de um lança bolos.

As fezes foram coletadas às $6,9,12,15$ e $18 \mathrm{~h}$, diariamente, até as 120 horas subseqüentes à aplicação das cápsulas. A excreção fecal dos animais durante os períodos experimentais foi obtida com base na relação:

Excreção fecal $(\mathrm{kg} / \mathrm{dia})=$ quantidade de indicador administrado $(\mathrm{mg})$ concentração do indicador nas fezes $(\mathrm{mg} / \mathrm{kg})$

A partir dos resultados obtidos de cromo fecal, foram confeccionadas as curvas de excreção para estimativas de consumo da dieta. As produções fecais, taxas de passagem no rúmen e os tempos de retenção foram calculados utilizando-se as variáveis obtidas dos modelos propostos por GROVUM e WILLIAMS (1973), QUIROZ et al. (1988) e POND et al. (1989).

O consumo da dieta total foi calculado pela fórmula: $($ consumo $=$ PF/ 1-DIVMS), sendo a produção fecal obtida a partir dos modelos de POND et al. (1989) e QUIROZ et al. (1988).

O consumo de matéria seca referente ao capim-

Rev. bras. zootec., 30(6S):2183-2191, 2001 
elefante foi calculado a partir da determinação da DIVMS do capim e da subtração das produções fecais calculadas para cada alimento, da excreção fecal total obtida nos modelos. Assim, na época seca foram medidas a ingestão do concentrado e da canade-açúcar, e ainda calculadas suas produções fecais utilizando-se as respectivas digestibilidades, obtidas em análises de laboratório.

$\mathrm{Na}$ época chuvosa, o consumo de matéria seca foi determinado da mesma forma, no entanto, subtraindo-se apenas a produção fecal do concentrado, da produção fecal total obtida a partir dos modelos.

As amostras das extrusas pré-secas e moídas foram analisadas para fibra em detergente neutro (FDN), fibra em detergente ácido (FDA) pelo método proposto por VAN SOEST (1965). Para as análises da digestibilidade in vitro da matéria seca (DIVMS), seguiram-se as recomendações de TILLEY e TERRY (1963). As determinações de cromo nas fezes foram feitas segundo o método de WILLIAMS et al. (1962), utilizando-se o espectrofotômetro de absorção atômica.

As estimativas dos parâmetros produções fecais, taxas de passagem no rúmen e os tempos de retenção foram obtidos pelos modelos propostos por GROVUM e WILLIAMS (1973), QUIROZ et al. (1988) e POND et al. (1989) como descrito a seguir:

a) GROVUM e WILLIAMS, (1973) - Modelo de dois compartimentos (idade independente nos dois compartimentos):

$$
\mathrm{y}=A \mathrm{e}^{-\mathrm{k} 1}(\mathrm{t}-\mathrm{T})-\mathrm{Ae}^{-\mathrm{k} 2(\mathrm{t}-\mathrm{T})} \text { para } \mathrm{t}>\mathrm{T}
$$

em que: $y=$ concentração do indicador; $t=$ tempo de amostragem; $\mathrm{T}$ = tempo de trânsito; $\mathrm{k} 1=$ estimativa da taxa de passagem do indicador no retículorúmen; $\mathrm{k}_{2}=$ estimativa da taxa de passagem do indicador no trato inferior; e = função exponencial (base do logaritmo natural $=2,7183$ )

b) QUIROZ et al. (1988) - Modelo de dois compartimentos (idade dependente e independente):

$$
y=C \phi\left[S^{2} e^{-k_{2} t}+e^{-\lambda t}\left(-S^{2}+S \lambda_{1} t\right)\right]
$$

em que: y = concentração do indicador nas fezes; $\mathrm{T}=$ tempo após a administração do indicador; $\mathrm{C} \phi=$ concentração inicial do indicador no trato gastrintestinal, assumindo mistura instantânea, no primeiro e segundo compartimentos, respectivamente; $\mathrm{K}_{2}=$ taxa de passagem da partícula, de idade independente, no segundo compartimento; $\lambda_{1}=$ taxa de

Rev. bras. zootec., 30(6S):2183-2191, 2001 passagem da partícula, de idade dependente no primeiro compartimento; $S=\lambda 1 /\left(\mathrm{k}_{2}-11\right)$; $\mathrm{T}=(\mathrm{t}-\mathrm{t}) ; \mathrm{t}=$ tempo entre $\mathrm{a}$ administração do indicador e o primeiro aparecimento nas fezes; $\mathrm{e}=$ função exponencial (base do logaritmo natural $=2,7183$ );

c) POND et al. (1989) - Modelo de um compartimento (idade dependente):

$$
\mathrm{y}=\left[\mathrm{K}_{0} \mathrm{~L}_{1}(\mathrm{t}-\tau) . \mathrm{e}-(\mathrm{L} 1(\mathrm{t}-\tau))\right] / 0,59635
$$

em que: $\mathrm{y}=$ concentração do indicador; $\mathrm{K}_{0}=$ concentração do indicador se este é misturado instantaneamente no compartimento; $\mathrm{L}_{1}=$ parâmetro de taxa de passagem dependente do tempo; $\tau=$ tempo decorrido da administração até o primeiro aparecimento do indicador nas fezes; $\mathrm{t}=$ tempo após a administração do indicador; $\mathrm{e}=$ função exponencial (base do logaritmo natural $=2,7183)$; taxa de passagem $(\mathrm{K} 1)=\mathrm{L}_{1} \mathrm{x}$ 0,59635 ; tempo médio de retenção $(\mathrm{TMR})=2 / \mathrm{L}_{1}+\mathrm{t}$; produção fecal $(\mathrm{g} /$ dia $)=\left[\right.$ indicador administrado $(\mu \mathrm{g}) / \mathrm{K}_{0}$ $\left.(\mu \mathrm{g} / \mathrm{g})] \times \mathrm{K}_{1} \times 24\right]$

Os parâmetros dos modelos estudados foram estimados usando-se o procedimento NLIN do programa SAS (SAS,1985). Foram fornecidos valores iniciais para os parâmetros, dentro do espaço paramétrico de cada um e segundo os valores esperados para os mesmos. Estes foram estimados inicialmente utilizando-se o método Marquardt.

Tais valores, assim estimados, foram utilizados novamente no NLIN, adotando-se para os ajustes das curvas de excreção o método de Gauss - Newton.

Os valores estimados nos três modelos para a taxa de passagem no rúmen, tempo médio de retenção no rúmen e no trato gastrintestinal, consumo de matéria seca total, consumo de capim-elefante, nos quatro meses experimentais e em duas doses de nitrogênio foram analisados, segundo o modelo:

$$
\begin{aligned}
\mathrm{Y}_{\mathrm{ijkl}}=\mu+\mathrm{B}_{\mathrm{i}}+\mathrm{D}_{\mathrm{j}}+\mathrm{E}_{\mathrm{k}}+\mathrm{M}_{1}+\mathrm{ED}_{\mathrm{kl}}+\mathrm{TM}_{\mathrm{jl}} \\
+\mathrm{EM}_{\mathrm{kl}}+\mathrm{e}_{\mathrm{ijkl}}
\end{aligned}
$$

em que: $\mathrm{Y}_{\mathrm{ijkl}}=$ valor para característica (consumo de MS total, consumo de MS de capim-elefante, taxa de passagem no rúmen, tempo médio de retenção no rúmem da iésima vaca que pastejou o piquete (bloco) no jéssima dose de nitrogênio na késsima época estimado pelo 1 éssimo modelo; $\mu=$ constante comum a cada observação; $\mathrm{B}_{\mathrm{i}}=$ efeito do bloco i ( $\mathrm{i}=1$ a 3 ); $\mathrm{D}_{\mathrm{j}}=$ efeito da dose de nitrogênio $\mathrm{j}(\mathrm{j}=1$ a 2$)$; $\mathrm{E}_{\mathrm{k}}=$ efeito do mês $\mathrm{k}$ ( $\left.\mathrm{k}=1 \mathrm{a} 4\right) ; \mathrm{Ml}=$ efeito do modelo $1(1=1$ a 3$) ; E_{\mathrm{kj}}=$ efeito da interação época x dose de nitrogênio; $\mathrm{TM}_{\mathrm{jL}}=$ efeito da interação dose de nitrogênio e tipo de modelo; $\mathrm{EM}_{\mathrm{kl}}=$ efeito da interação época $\mathrm{x}$ modelo; $\mathrm{e}_{\mathrm{ijkl}}=$ erro residual aleatório 
O experimento constituiu-se de um fatorial $2 \times 4 \times 3$ (duas doses de N; quatro meses; três modelos) cujos fatores foram distribuídos no delineamento em blocos casualizados com três repetições, e para a comparação entre médias utilizou-se o teste de NewmanKeuls a 5\% de probabilidade. A análise de variância e a aplicação dos testes de significância foram feitas no programa SAS (1985).

\section{Resultados e Discussão}

A disponibilidade de matéria seca por hectare (MS/ha) de forragem, assim como o consumo de matéria seca do capim-elefante (CCE) e da dieta total (CMST) estimada pelos diferentes modelos não apresentaram diferenças $(\mathrm{P}>0,05)$ entre os níveis de nitrogênio utilizados na pastagem de capim-elefante, contudo, detectou-se efeito $(\mathrm{P}<0,05)$ para os diferentes meses estudados.

Para a disponibilidade de matéria seca por hectare (MS/ha) de forragem, foram observados valores de 472,$7 ; 1456,6 ; 2242,5$ e 2354,8 kg/ha de julho, outubro, janeiro e março, respectivamente (Tabela 1).

AROEIRA et al. (1999) observaram menores valores de disponibilidade para o capim elefante de $1.939 \mathrm{~kg}$ de $\mathrm{MS} / \mathrm{ha}$, entretanto com um consumo médio de $3,5 \%$ PV e uma taxa de lotação de 4,5 vacas/ha.

O capim-elefante, como a maioria das forrageiras tropicais, caracteriza-se por obter 70 a $80 \%$ da produção concentrada na época das chuvas. Esta estacionalidade de produção forrageira é atribuída às

Tabela 1 -Disponibilidade média $(\mathrm{kg} / \mathrm{ha})$ de matéria seca (MS) do capim-elefante, em função dos meses avaliados

Table 1 - Average dry matter (DM) availability $(\mathrm{kg} / \mathrm{ha})$ of elephantgrass in four seasons of the year

\begin{tabular}{lc}
\hline $\begin{array}{l}\text { Meses } \\
\text { Months }\end{array}$ & $\begin{array}{c}\text { Disponibilidade de MS (kg/ha) } \\
\text { DM availability }(\mathrm{kg} / \mathrm{ha})\end{array}$ \\
\hline Julho & $472,7 \pm 87,5^{\mathrm{c}}$ \\
July & $1.455,6 \pm 107,2^{\mathrm{b}}$ \\
Outubro & \\
October & \\
Janeiro & $2.242,5 \pm 107,2^{\mathrm{a}}$ \\
January & $2.354,8 \pm 107,2^{\mathrm{a}}$ \\
Março & 24,7 \\
March &
\end{tabular}

Médias seguidas de letras diferentes na mesma coluna diferem $(P<0,05)$ pelo teste de Newman-Keuls.

Values within column with different letters significantly differ $(P<.05)$ by Newman-Keuls test. baixas precipitações, pouca luminosidade e baixas temperaturas que ocorrem no período do inverno. DERESZ (2001) observou, na mesma região do presente estudo, disponibilidades variando de $1850 \pm 282$ a $721 \pm 118 \mathrm{~kg}$ de $\mathrm{MS} / \mathrm{ha}$ de novembro a junho, no entanto utilizando uma taxa de lotação de 4,5 vacas $/$ ha e adubado com doses de nitrogênio $(200 \mathrm{~kg} / \mathrm{ha})$ inferiores ao do presente estudo.

Os teores de FDN e FDA da extrusa apresentaram valores elevados de julho a março (Tabela 2). Isto deve-se às condições ambientais favoráveis ao crescimento do capim-elefante, principalmente em janeiro e março, como disponibilidade hídrica, temperaturas elevadas e alta luminosidade, promovendo assim maior alongamento do caule e, conseqüentemente, maior lignificação da parede celular. Os menores teores de FDN observados em julho e outubro devem-se possivelmente à baixa disponibilidade de forragem, levando então os animais a exercerem uma maior seleção por folhas novas das recentes brotações ocorridas nas touceiras de capim-elefante que representam uma fração menos fibrosa comparada com a planta inteira e com o caule.

Quanto à DIVMS, observou-se que os valores encontrados em julho e outubro foram superiores

Tabela 2 - Teores médios de fibra em detergente neutro (FDN), fibra em detergente ácido (FDA) e digestibilidade in vitro da matéria seca (DIVMS) das extrusas de capim-elefante em quatro épocas do ano

Table 2 - Means of neutral detergente fiber (NDF), acid detergente fiber (ADF) and in vitro dry matter digestibility (IVDMD) of elephantgrass extrusa in four seasons of the year

\begin{tabular}{lccc}
\hline Meses & \multicolumn{3}{c}{$\begin{array}{c}\text { Variáveis (\% da MS) } \\
\text { Variables (\% of DM) }\end{array}$} \\
\cline { 2 - 4 } & FDN & $\begin{array}{c}\text { FDA } \\
\text { ADF }\end{array}$ & $\begin{array}{c}\text { DIVMS } \\
\text { NDF }\end{array}$ \\
\cline { 2 - 4 } & $65,59^{\mathrm{b}}$ & $34,89^{\mathrm{b}}$ & $67,48^{\mathrm{a}}$ \\
\hline $\begin{array}{l}\text { Julho } \\
\text { Ouly }\end{array}$ & $64,08^{\mathrm{b}}$ & $33,97^{\mathrm{b}}$ & $65,72^{\mathrm{a}}$ \\
$\begin{array}{l}\text { Octubro } \\
\text { Janeiro }\end{array}$ & $67,33^{\mathrm{ab}}$ & $39,63^{\mathrm{a}}$ & $59,61^{\mathrm{b}}$ \\
$\begin{array}{l}\text { January } \\
\text { Março }\end{array}$ & $69,47^{\mathrm{a}}$ & $38,95^{\mathrm{a}}$ & $61,08^{\mathrm{b}}$ \\
$\begin{array}{l}\text { March } \\
\text { CV(\%) }\end{array}$ & 3,7 & 2,9 & 3,1 \\
$\begin{array}{l}\text { Média } \\
\text { Average }\end{array}$ & 66,6 & 36,9 & 63,5
\end{tabular}

Average

Médias seguidas de letras diferentes na mesma coluna diferem entre si $(P<0,05)$, pelo teste de Newman-Keuls.

Values within column with different letters differ significantly by Newman-Keuls test $(P<.05)$.

Rev. bras. zootec., 30(6S):2183-2191, 2001 
$(\mathrm{P}<0,05)$ aos registrados em janeiro e março, que por sua vez não diferiram entre si $(\mathrm{P}>0,05)$, como pode ser observado na Tabela 2. Os valores mais elevados, registrados em julho e outubro, podem estar associados aos menores $(\mathrm{P}<0,05)$ teores de FDA, verificados nestes períodos, uma vez que, segundo VAN SOEST (1965), este componente está consistentemente correlacionado com a digestibilidade.

Os consumos de MS estimados pelos modelos de QUIROZ et al. (1988) e POND et al. (1989) foram semelhantes entre si $(\mathrm{P}>0,05)$. Foram observados consumos médios de $8,5 \mathrm{~kg} / \mathrm{vaca} / \mathrm{dia}$ ou $1,7 \%$ do peso vivo (PV) para o capim-elefante e de $13,3 \mathrm{~kg} / \mathrm{vaca} /$ dia ou 2,6\% PV para a dieta total (Tabela 3). Consumos de pasto ligeiramente superiores de 14,9 kg/vaca/dia ou 2,1\% PV foram descritos por DERESZ et al. (1997) e AROEIRA et al. (1999), estimados com auxílio do óxido crômico, em vacas mestiças com produção de leite média de 11,4 kg/vaca/dia e $483 \mathrm{~kg}$ de PV, em pastagens suplementadas, numa taxa de lotação de 4,5 vacas/ha. Já SOARES et al. (1999), nas mesmas condições experimentais do presente trabalho, observaram, em quatro épocas de consumo, médias inferiores às estimadas no presente estudo (6,0 kg $/ \mathrm{vaca} /$ dia e $1,1 \%$ do PV), utilizando o modelo de QUIROZ et al. (1988).

Os valores encontrados para o consumo de MS, no presente trabalho, podem ter sido menores, em relação aos dados obtidos com óxido crômico, devido aos seguintes fatores: baixa qualidade da pastagem,

Tabela 3 - Consumo de matéria seca (MS) do capim-elefante e de matéria seca da dieta total de vacas mestiças em lactação, estimados pelos modelos de POND et al. (1989) e QUIROZ et al. (1988)

Table 3 - Elephantgrass dry matter intake and total diet dry matter intake of lactating crossbred cows, estimated by models of POND et al. (1989) and QUIROZ et al. (1988)

\begin{tabular}{|c|c|c|c|c|}
\hline \multirow[t]{3}{*}{$\begin{array}{l}\text { Modelo } \\
\text { Model }\end{array}$} & \multicolumn{4}{|c|}{$\begin{array}{l}\text { Consumo de MS } \\
\text { Dry matter intake }\end{array}$} \\
\hline & \multicolumn{2}{|c|}{$\begin{array}{c}\text { Capim-elefante } \\
\text { Elephantgrass }\end{array}$} & \multicolumn{2}{|c|}{$\begin{array}{c}\text { Dieta total } \\
\text { Total diet }\end{array}$} \\
\hline & $\begin{array}{c}(\mathrm{kg} / \mathrm{dia}) \\
(\mathrm{kg} / \text { day })\end{array}$ & $\begin{array}{l}(\% \mathrm{PV}) \\
(\% B W)\end{array}$ & $\begin{array}{c}\text { ( kg/dia) } \\
(\mathrm{kg} / \text { day })\end{array}$ & $\begin{array}{l}(\% \mathrm{PV}) \\
(\% B W)\end{array}$ \\
\hline POND et al.(1989) & 7,75 & 1,5 & 12,55 & 2,46 \\
\hline QUIROZ et al. (1988) & ) 9,24 & 1,83 & 13,98 & 2,77 \\
\hline $\mathrm{CV}(\%)$ & 72,3 & 45,2 & 48,3 & 31,3 \\
\hline $\begin{array}{l}\text { Média } \\
\text { Average }\end{array}$ & 8,5 & 1,7 & 13,3 & 2,6 \\
\hline
\end{tabular}

taxa de lotação utilizada (6 vacas/ha) e baixa recuperação do cromo mordente, observadas nas concentrações de cromo nas fezes.

Os consumos de matéria seca da dieta total (CMST) e do capim-elefante (CCE) nos diferentes meses (julho, outubro, janeiro e março) não apresentaram diferenças significativas $(\mathrm{P}>0,05)$ entre modelos. Apesar da grande diferença numérica, não foram detectadas diferenças significativas $(\mathrm{P}>0,05)$ no consumo de MS de capim-elefante de 7,33 e $4,80 \mathrm{~kg} / \mathrm{vaca} /$ dia em julho, 7,10 e $9,10 \mathrm{~kg} / \mathrm{vaca} / \mathrm{dia}$ em outubro, 8,47 e $9,32 \mathrm{~kg} / \mathrm{vaca} / \mathrm{dia}$ em janeiro e 14,30 e $7,52 \mathrm{~kg} / \mathrm{vaca} /$ dia em março (Tabela 4) estimados, respectivamente, pelos modelos de QUIROZ et al. (1988) e POND et al. (1989). Isto pode ter ocorrido em função dos elevados coeficientes de variação (CV\%) observados nestas estimativas, principalmente para os valores de consumo do capim-elefante, que são gerados indiretamente por modelos distintos e com diferentes variáveis, além da própria individualidade dos animais e das diferentes épocas avaliadas, causando grandes variações nos dados obtidos, mas que são geralmente observados em trabalhos desta natureza.

Tabela 4 - Consumo de matéria seca da dieta total (CMST) e de matéria seca do capim-elefante (CCE), expressos em kg/dia, em quatro meses do ano, segundo os modelos propostos por QUIROZ et al. (1988) e POND et al. (1989)

Table 4 - Total dry matter intake (DMI) and elephantgrass dry matter intake (EDMI) according to the month of the year, estimated by models proposed by QUIROZ et al. (1988) and POND et al. (1989)

\begin{tabular}{|c|c|c|c|c|}
\hline \multirow[t]{3}{*}{$\begin{array}{l}\text { Meses } \\
\text { Months }\end{array}$} & \multicolumn{4}{|c|}{$\begin{array}{c}\text { Modelos } \\
\text { Models }\end{array}$} \\
\hline & \multicolumn{2}{|c|}{$\begin{array}{c}\text { QUIROZ et al. } \\
(1988)\end{array}$} & \multicolumn{2}{|c|}{$\begin{array}{c}\text { POND et al. } \\
(1989)\end{array}$} \\
\hline & $\begin{array}{c}\text { CMST } \\
\text { (kg/dia) } \\
\text { DMI } \\
\text { (kg/day) }\end{array}$ & $\begin{array}{c}\mathrm{CCE} \\
(\mathrm{kg} / \mathrm{dia}) \\
E D M I \\
(\mathrm{~kg} / \text { day })\end{array}$ & $\begin{array}{c}\text { CMST } \\
\text { (kg/dia) } \\
\text { DMI } \\
(\mathrm{kg} / \text { day })\end{array}$ & $\begin{array}{c}\mathrm{CCE} \\
(\mathrm{kg} / \mathrm{dia}) \\
E D M I \\
(\mathrm{~kg} / \text { day }\end{array}$ \\
\hline $\begin{array}{l}\text { Julho } \\
\text { July }\end{array}$ & 15,42 & 7,33 & 12,33 & 4,80 \\
\hline $\begin{array}{l}\text { Outubro } \\
\text { October }\end{array}$ & 14,59 & 7,10 & 15,38 & 9,10 \\
\hline $\begin{array}{l}\text { Janeiro } \\
\text { January }\end{array}$ & 10,19 & 8,47 & 11,04 & 9,32 \\
\hline $\begin{array}{l}\text { Março } \\
\text { March }\end{array}$ & 15,92 & 14,30 & 9,15 & 7,52 \\
\hline $\mathrm{CV}(\%)$ & 48,3 & 72,3 & 48,3 & 72,3 \\
\hline $\begin{array}{l}\text { Média } \\
\text { Average }\end{array}$ & 14,0 & 9,3 & 11,9 & 7,7 \\
\hline
\end{tabular}

Rev. bras. zootec., 30(6S):2183-2191, 2001 
Entretanto, os resultados estimados pela média de ambos os modelos detectaram diferenças significativas $(\mathrm{P}<0,05)$ entre os meses para o consumo total. Os consumos médios de MS da dieta total foram mais elevados $(\mathrm{P}<0,05)$ no mês de outubro $(14,99 \mathrm{~kg} / \mathrm{vaca} /$ dia), em relação aos consumos observados em janeiro (10,61 kg/vaca/dia). Os resultados obtidos em julho e março (13,87 e 12,53 kg/vaca/dia, respectivamente) foram semelhantes $(\mathrm{P}>0,05)$ entre si e aos demais meses (Tabela 5). Por outro lado, o consumo médio de MS do capim-elefante foi superior $(\mathrm{P}<0,05) \mathrm{em}$ março $(10,91 \mathrm{~kg} / \mathrm{vaca} / \mathrm{dia})$ em relação ao mês de julho $(6,07 \mathrm{~kg} / \mathrm{vaca} / \mathrm{dia})$. Os resultados obtidos em outubro e janeiro $(8,14$ e $8,90 \mathrm{~kg} / \mathrm{vaca} /$ dia, respectivamente $)$ foram semelhantes $(\mathrm{P}>0,05)$ entre si e aos demais meses. As diferenças observadas no consumo de MS da dieta total entre os meses se justificam, principalmente pela participação do capim-elefante na dieta, que foi em média de 26 e $84 \%$ da matéria seca total, em julho/outubro e janeiro/março, respectivamente. Nos dois primeiros meses, a mistura cana-de-açúcar e uréia participou em 58\%, devido à baixa disponibilidade de forragem neste período, além da suplementação concentrada que contribuiu com $16 \%$ da dieta total durante todo o período experimental.

Tabela 5 - Consumo médio de matéria seca da dieta total (CMST) e de matéria seca do capim-elefante (CCE) estimado pelos dois modelos, nos diferentes meses do ano

Table 5 - Average of total dry matter intake (DMI) and elephantgrass dry matter intake (EDMI), estimated by the two models, according to the month of the year

\begin{tabular}{lcc}
\hline $\begin{array}{l}\text { Meses } \\
\text { Months }\end{array}$ & $\begin{array}{c}\text { CMST (kg/dia) } \\
D M I(k g / \text { day })\end{array}$ & $\begin{array}{c}\text { CCE }(\mathrm{kg} / \text { dia }) \\
E D M I(k g / \text { day })\end{array}$ \\
\hline $\begin{array}{l}\text { Julho } \\
\text { July }\end{array}$ & $13,87^{\mathrm{ab}}$ & $6,07^{\mathrm{b}}$ \\
$\begin{array}{l}\text { Outubro } \\
\text { October }\end{array}$ & $14,99^{\mathrm{a}}$ & $8,14^{\mathrm{ab}}$ \\
Janeiro & $10,61^{\mathrm{b}}$ & \\
January & $12,53^{\mathrm{ab}}$ & $8,90^{\mathrm{ab}}$ \\
$\begin{array}{l}\text { Março } \\
\text { March }\end{array}$ & & $10,91^{\mathrm{a}}$ \\
CV(\%) & 49,4 & 73,9 \\
Média & 13,0 & 8,5 \\
Average & &
\end{tabular}

Médias seguidas de letras diferentes na mesma coluna diferem $(P<0,05)$, pelo teste de Newman-Keuls.

Values within column with different letters differ $(P<.05)$ by Newman-Keuls test.
Com relação aos modelos utilizados, os valores observados para os consumos de MS estão diretamente ligados às estimativas de excreção fecal total obtidas por meio destes, conseqüentemente influenciando os consumos, possivelmente devido à perda de indicador na dosificação (COLUCCI, 1984), na marcação da fibra com o cromo, como também pelo tamanho da fibra (QUIROZ et al., 1988), além do ajustamento dos modelos (PRANGE et al., 1982).

As taxas de passagem no rúmen, os tempos médios de retenção no rúmen e no trato gastrintestinal apresentaram diferenças $(\mathrm{P}<0.05)$ entre os modelos (Tabela 6). As taxas de passagem, 3,0\%/hora estimada pelos modelos de GROVUM e WILLIAMS (1973) e 2,8 \%/hora por POND et al. (1989), foram semelhantes entre si $(\mathrm{P}>0,05)$. Entretanto, a estimada pelo modelo de QUIROZ et al. (1988), de 4,1\%/hora, foi superior às demais $(\mathrm{P}<0,05)$.

O valor médio de $4,1 \% / \mathrm{h}$, encontrado para a taxa de passagem ruminal obtido pelo modelo de QUIROZ et al. (1988), é mais elevado que os $3 \% / \mathrm{h}$, obtidos por VIEIRA (1995) para o capim-elefante colhido aos 61 e 145 dias. Por outro lado, os valores obtidos pelos modelos de GROVUM e WILLIAMS (1973) e POND et al. (1988) foram semelhantes aos encontrados por VIEIRA (1995) utilizando o modelo de GROVUM e WILLIAMS (1973).

A taxa de passagem no rúmen é uma variável de grande importância, pois determina o fluxo de digesta pelo trato gastrintestinal que, no caso de forrageiras tropicais, detém valores baixos devido principalmente ao alto teor de fibra. A cinética de trânsito ou passagem refere-se ao fluxo de resíduos não digeridos do alimento ao longo do trato digestivo e pelos resultados observados do consumo de matéria seca e das taxas de passagem obtidas nos diferentes modelos, embora sem muitas variações, podem ter sido influenciadas por vários fatores. Segundo MERTENS e ELY (1982), estes parâmetros estão condicionados principalmente pelo nível de consumo, pela forma física da dieta, pelas diferenças na ruminação existentes entre animais, pelo tipo de indicador usado na determinação da curva de excreção fecal, pela proporção volumoso: concentrado e por fatores climáticos (FAICHNEY, 1993).

Os tempos de retenção ruminal (TRR) da fibra do capim-elefante (Tabela 6) para os três modelos superaram o valor de 19,6 horas, obtido por GOMES (1991), para o feno de capim-elefante cv. Napier.

O tempo médio de retenção no trato gastrintestinal para os modelos de GROVUM e WILLIAMS (1973)

Rev. bras. zootec., 30(6S):2183-2191, 2001 
e POND et al. (1989) foram, respectivamente, de 82,54 e 83,24 horas (Tabela 6). Resultados semelhantes foram reportados por FURLAN (1998), estimados pelo modelo de POND et al. (1989). Observaram-se valores de 86,33 e 75,71 horas, respectivamente, para vacas da raça Gir e Girolanda, sob pastejo de capim coast-cross. Tempo médio de retenção no trato gastrintestinal mais elevado (92.4 horas), estimado pelo modelo de POND et al. (1989) foi observado por SALMAN, (1999) em vacas mestiças, sob pastejo de capim-tanzânia.

MAMBRINI et al. (1988), comparando diferentes modelos na determinação do tempo médio de retenção total no trato gastrintestinal, observou valor semelhante (56,3 horas) aos obtidos pelo modelo de QUIROZ et al. (1988) no presente estudo.

O tempo de retenção no rúmen representa o tempo em que o alimento permanece no rúmen sofrendo a ação dos microorganismos para a redução do tamanho das partículas alimentares, sendo um parâmetro inversamente correlacionado com a taxa de passagem no rúmen $\left(\mathrm{TRR}=1 / \mathrm{K}_{1}\right)(\mathrm{GROVUM} \mathrm{e}$ WILLIAMS, 1973), além de ser influenciado pelas

Tabela 6 - Taxas de passagem no rúmen $\left(\mathrm{K}_{1}\right)$ e tempos médios de retenção no rúmen (TRR) e no trato gastrintestinal (TRT) de vacas mestiças em pastagem de capim-elefante estimados pelos modelos de GROVUM e WILLIAMS (1973), QUIROZ et al. (1988) e POND et al. (1989)

Table 6 - Rate of passage in the rumen $\left(k_{1}\right)$ and mean retention time in rumen (RRT) and in the total gastrointestinal tract (TRT), of crossbred cows in elephantgrass pasture estimated by the models of GROVUM and WILLIAMS (1973), QUIROZ et al. (1988) and POND et al. (1989)

\begin{tabular}{lccl}
\hline Modelo & \multicolumn{3}{c}{$\begin{array}{c}\text { Parâmetros } \\
\text { Parameters }\end{array}$} \\
\cline { 2 - 4 } & $\mathrm{K}_{1}\left(\mathrm{~h}^{-1}\right)$ & TRR $(\mathrm{h})$ & $\mathrm{TRT}(\mathrm{h})$ \\
& $K_{1}\left(h^{-1}\right)$ & $R R T(h)$ & $T R T(h)$ \\
\hline GROVUMe & $0,030^{\mathrm{b}}$ & $51,10^{\mathrm{a}}$ & $82,54^{\mathrm{a}}$ \\
WILLIAMS (1973) & & & \\
POND et al.(1989) & $0,028^{\mathrm{b}}$ & $38,04^{\mathrm{b}}$ & $83,24^{\mathrm{a}}$ \\
QUIROZ et al.(1988) & $0,041^{\mathrm{a}}$ & $35,10^{\mathrm{b}}$ & $58,30^{\mathrm{b}}$ \\
CV(\%) & 64,6 & 59,4 & 36,4 \\
Média & 0,033 & 41,4 & 74,7 \\
Average & & & \\
\hline
\end{tabular}

Médias seguidas de letras diferentes na mesma coluna diferem $(P<0,05)$, pelo teste de Newman-Keuls.

Values within column with different letters differ by Newman-Keuls test $(P<.05)$. concentrações de fibra na dieta (FORBES, 1995) e dependente do limite físico do animal (JUNG e ALLEN, 1995).

MOORE et al. (1992), estudando estimativas da cinética da digesta e testando cinco modelos nãolineares de idade dependente, observaram que vários modelos podem ser estudados para determinação destes parâmetros, entretanto, o mais adequado será aquele que apresentar melhor ajuste aos dados utilizados e menor variação entre os dados obtidos.

Como os modelos estudados apresentavam variáveis para estimativas de parâmetros diferentes, não puderam ser comparados pela utilização de dispersão dos valores e relacionados pelas suas respectivas regressões ou erros experimentais; no entanto, acredita-se que modelos de dois compartimentos e de idade dependente, em comparação aos modelos de idade independente e de um compartimento, sejam mais precisos nas estimativas da cinética do trato gastrintestinal de ruminantes, utilizando-se alimentos fibrosos.

No presente trabalho, acredita-se também que a baixa recuperação do indicador nas fezes possa ter sido causada pela falta de condições de equilíbrio deste no trato gastrintestinal (PIAGGIO et al., 1991), influenciando os parâmetros estimados pelos modelos, assim como a qualidade da dieta ingerida, além da necessidade de maior período de coletas de fezes ao longo do dia, o que contribuiria para melhor caracterização de uma curva de excreção fecal, induzindo a estimativas de parâmetros mais eficientes pelos modelos.

\section{Conclusões}

Os parâmetros estimados pelos modelos podem ter sido influenciados pela baixa recuperação do indicador nas fezes, possivelmente devido à perda de indicador na dosificação, principalmente na marcação da fibra com o cromo, como também pelo tamanho da fibra, além do ajustamento individual de cada modelo.

O modelo de QUIROZ et al. (1988) parece ser o mais adequado por apresentar dois compartimentos com idade dependente para o primeiro compartimento e independente para o segundo, acreditando-se que, por isto, este seja mais preciso nas estimativas da cinética do trato gastrintestinal de ruminantes, utilizando-se alimentos fibrosos, além dos resultados obtidos estarem mais próximos ao observado na literatura. 


\section{Referências Bibliográficas}

AROEIRA, L.J.M., LOPES, F.C.F., DERESZ, F. et al. 1999. Pasture availability and dry matter intake of lactating crossbred cows grazing elephant grass (Pennisetum purpureum, Schum.) Anim. Feed Scie. and Tech., 78:313-324.

AROEIRA, L.J.M., LOPES, F.C., MALDONADO-VASQUEZ, H. et al. Disponibilidade e consumo do capim-elefante (Pennisetum purpureum, Schum.) sob pastejo de vacas em lactação. In: REUNIÃO ANUAL DA SOCIEDADE BRASILEIRA DE ZOOTECNIA, 33., Fortaleza, 1996. Anais... Fortaleza: SBZ, 1996.

COLUCCI, P.E. Comparative digestion and digesta kinetics in sheep and cattle. Canadá, 1984. 166p. (Thesis) University of Guelph. 1984.

DERESZ, F.200. Produção de leite de vacas mestiças Holandês $\mathrm{x}$ Zebu em pastagem de capim-elefante, manejada em sistema rotativo com ou sem suplementação durante a época das chuvas. R. Soc. Bras. Zootec., 30(1):197-204.

DERESZ, F., OLIVEIRA, J.S., CAMPOS, O.F. Produção de leite e consumo de vacas mestiças Holandês $\mathrm{x} Z$ Zebu recebendo capim-elefante picado, com ou sem concentrado. In REUNIÃO ANUAL DA SOCIEDADE BRASILEIRA DE ZOOTECNIA, 34, 1997, Juiz de Fora. Anais... Juiz de Fora: SBZ, 1997. p.246-248.

DHANOA, M.S., SIDDONS,R.C., FRANCE, J. et al. 1985. A multicompartimental model to describe marker excretion patterns in ruminant feaces. Brit. J. Nut., 53:663-671.

ELLIS, W.C., MATIS, J.H., LASCANO, C. 1979. Quantitating ruminal turnover. Fed. Proc., 38:2702.

FAICHNEY, G.J. 1993. Digesta flow. In: FORBES, J.M., FRANCE, J. (Eds.) Quantitative of ruminant digestion and metabolism. Commonwealth Agricultural Bureaux, Cambridge University Press. p.53-85.

FORBES, J. M. 1995.Voluntary food intake and diet selection in farms animals. Walingford: Cab International. 532p.

FURLAN, C.L. Estimativa da disponibilidade, composição da extrusa e consumo de matéria seca de vacas em lactação Gir e Girolanda sob pastejo intensivo em capim coast cross (Cynodon dactylon (L.) Pears.) var. coast cross. Jaboticabal, SP: FCAV-UNESP, 1998. 76p. Dissertação (Mestrado em Zootecnia) - Universidade Estadual Paulista, 1998.

GOMES, B.V. Influência das características químicas e físicas das forrageiras sobre o consumo, degradação e cinética da digesta ruminal. Viçosa, MG: UFV, 1991. 115p. Tese (Doutorado em Zootecnia) - Universidade Federal de Viçosa, 1991.

GROVUM, W.L., WILLIAMS, V.J. 1973. Rate of passage of digesta in sheep. 4. Passage of marker through the alimentary tract and the biological relevance of rate-constants derived from the changes in concentrationof marker in faeces. Br. J. Nutr., 30(1):313-329.

JUNG, H.G., ALLEN, M.S. 1995. Characteristics of plant cell walls affecting intake and digestibility of forages by ruminants. J. Anim. Sci., 73(11):2774-2790.

LUGINBUHL, J.M., POND, K.L., BURNS, J.C. et al. Computer interface system to monitor the ingestive and ruminanting behavior of grazing ruminants. In: GRAZING LIVESTOCK NUTRITION CONFERENCE, 2, 1991, Steamboat Springs. Anais... Steamboat Springs, Colorado, 1991. p.177.

MAMBRINI, M., PEYRAUD, J.L., RULQUIN, H. 1988. Comparaison de différentes méthodes de calcul du temps de séjour des résidus alimentaires dans l' ensemble du tube digestif chez la vache laitière. Reprod. Nutr. Dévelop., 28(1):149-150.

Rev. bras. zootec., 30(6S):2183-2191, 2001
MERTENS, D.R., ELY, L.O. 1982. Relationship of rate and extent of digestion to forage utilization - a dynamic model evaluation. J. Anim. Sci., 54:895-905.

MOORE, J.A., POND, K.R., POORE, M.H. et al. 1992. Influence of model and marker on digesta kinetic estimates for sheep. J. Anim. Sci., 70(12):3528-3540.

OWENS, F.N., HANSON, C.F. 1992. External and internal markers for appraising site and extent of digestion in ruminants. J. Dai. Sci., 75:2605-2617.

PIAGGIO, L.M., PRATES, E.R., PIRES, F.F. et al. 1991. Avaliação do cromo mordente como indicador externo da produção fecal. R. Soc. Bras. Zootec., 20(3):313-318.

POND, K.R., ELLIS, W.C., MATIS, J.H., DESWYSEN, A. G. 1989. Passage of chromium-mordanted and rare earth-labeled fiber: time dosing kinetics. J. Anim. Sci., 67:1020-1028.

POND, K.R., BURNS, J.C., FISHER, D.S., et al. Intake monitoring systems: eletronics markers. Tulsa, Oklahoma: Word Wide Web, USA, 1994. p.1-15. (USDA)

PRANGE, R.W., JORGENSEN, N.A., SATTER, L.D. 1982. Rate of passage calculations based on duodenal or fecal collections sites. J. Dairy Sci., 65(1):145.

QUIROZ, R.A., POND, K.R., TOLLEY, E. A. et al. 1988. Selection among nonlinear models for rate of passage studies in ruminants. J. Anim. Sci., 66:2977-2986.

SAS. 1985. Statistics Analitical System, sas users guide: statistics, Institute Inc., Cary: USA. 965p.

SALMAN, A.K.D. Degradabilidade in situ e consumo voluntário do capim Tanzânia (Pannicum maximum, J.cv. Tanzânia), sob pastejo, por vacas em lactação. Jaboticabal, SP:UNESP, 1999. 62p. Dissertação (Mestrado em Zootecnia) Faculdade de Ciências Agrárias e Veterinárias, Universidade Estadual Paulista, 1999.

SEONE, J.R. Selected topics on intake and utilization of forages by cattle In: ANIMAL SCIENCE RESEARCH AND DEVELOPMENT-MOVING TOWARD A NEW CENTURY, Ottawa, Ontario, Canada, 1995. p.243-261.

SOARES, J.P.G.; AROEIRA, L.J.M.; PEREIRA, O.G.; et al. 1999. Capim-elefante (Pennisetum purpureum Schum.), sob duas doses de nitrogênio. consumo e produção de leite. $R$. Soc. Bras. Zootec., 28(4):889-897.

TILLEY, J.M.A., TERRY, R.A. 1963. A two stages techinique for the in vitro digestion of forage crops. J. Br. Grassl. Soc. 18(2):104-111.

VAN SOEST, P.J. 1965. Symposium on factors influencing the voluntary intake of herbage by ruminants: Voluntary intake relation to chemical composition and digestibility. J. Anim. Sci., 24(3):834-44.

VIEIRA, R.A.M.Modelos matemáticos paraestimativa deparâmetros da cinética de degradação do capim-elefante (Pennisetum purpureum, Schum., cv. Mineiro) em diferentes idades de corte. Viçosa, MG: UFV, 1995. 68p. Dissertação (Mestrado em Zootecnia) - Universidade Federal de Viçosa, 1995.

WILliAMS, C.H., DAVID, D.J., IISMAA, O. 1962. The determination of chromic oxide in faeces samples by atomic absorption. J. Agric. Sci., 59:381-385.

Recebido em: 05/10/00 Aceito em: 20/08/00 